\title{
Medical Comorbidities in Autism: Challenges to Diagnosis and Treatment
}

\author{
Margaret L. Bauman \\ Department of Neurology, Department of Pediatrics, and Lurie Center LADDERS program, Massachusetts General Hospital, \\ and Department of Neurology, Harvard Medical School, Boston, Massachusetts
}

Summary: Ever since its original description by Leo Kanner in 1943, autism has been generally defined by its clinical characteristics and core symptoms that include impaired social skills, isolated areas of interest, and delayed and disordered language. Over time, it has become apparent that autism is a heterogeneous disorder with regard to its clinical presentation, etiology, underlying neurobiology, and degree of severity. As a result, the termed diagnosis of autism spectrum disorders (ASDs) has come into common usage. With advancements in clinical care, there has come the appreciation that many ASD children, adolescents, and adults may have medically relevant disorders that may negatively impact their developmental progress and behavior, but which frequently go undetected. Many of these medical conditions are treatable, often resulting in improved developmental gains and quality of life for the patient and family. In addition, the possibility exists that some of these medical conditions may suggest the presence of important genetic and/or biologic markers, which, if identified, can refine our ability to be more precise in categorizing clinical and genetic subtypes within the autism spectrum. Key Words: Autism, medical conditions, atypical behaviors, diagnosis, management.

\section{INTRODUCTION}

Infantile autism is a behaviorally defined disorder first described by Leo Kanner ${ }^{1}$ in 1943. Although infantile autism is now recognized as being clinically, etiologically, and biologically heterogeneous, it is generally agreed that the core features of the disorder consist of a triad of characteristics, including impaired social interaction, delayed and disordered language, and isolated areas of interest. ${ }^{2}$ Variable additional features can include poor eye contact, stereotypic and repetitive behaviors, sensory processing dysfunction, and an insistence on sameness. Symptoms can range from relatively subtle and mild to very severe. Some level of cognitive impairment has been reported. Until recently, it was generally believed that $75 \%$ of those affected with autism functioned in the mentally retarded range. However, more current data suggests that fewer than half of those affected have a significant cognitive deficit. ${ }^{3}$ Although it was once believed that autistic persons had no impairment of motor skills, a growing body of research sug-

Address correspondence and reprint requests to: Margaret L. Bauman, M.D., Lurie Center LADDERS, 1 Maguire Road, Lexington, MA 02421. E-mail: drb@ ladders.org. gests that hypotonia is common and may be associated with deficits in both fine and gross motor skills. ${ }^{4}$ Individuals with non-syndromic or "essential" autism and with few, if any, dysmorphic features are generally considered to be attractive in appearance. Measurements of head circumference in childhood has suggested that approximately $20 \%$ of autistic children show a frank macrocephaly of greater than $98 \%$ for age and sex. ${ }^{5}$ Numerous epidemiologic studies have provided evidence for a genetic basis for autism. Linkage studies have identified a number of candidate genes of promise; however, despite modern technology, approximately only $6 \%$ to $15 \%$ of individuals with autism will be found to have an identifiable genetic diagnosis. ${ }^{6-8}$ Current studies suggest a multifactorial inheritance, including genetic heterogeneity with multiple major gene effects, possible contributing environmental effects, and physiologically linked processes with multiple genes. Thus, autism is now considered a heterogeneous disorder, clinically, neurobiologically, and etiologically, resulting in the now common usage of the termed diagnosis of autism spectrum disorders (ASDs).

For the most part, autism research has been largely devoted to the assessment of and understanding of many of the clinical characteristic features of the disorder, 
generally aspects of language patterns, cognitive skills, behavioral manifestations, social interaction, and information processing, and how these features may relate to the underlying gross and microscopic anatomy, neurochemistry, connectivity of the brain, and potential genetic factors. Although we still have much to learn, we have expanded our knowledge regarding the underlying neurobiological mechanisms related to the autistic brain during the past 25 years. However, we have acquired very little knowledge of any other organ systems that may be important in this disorder. Much of this relative lack of information may relate to the fact that individuals with ASD, especially children, can be difficult to examine, often due to poor cooperation and difficult office behavior. Other limitations include the fact that many ASD individuals are nonverbal or hypo-verbal, and many of these patients have deficits in sensory processing, which are features that prevent them from reporting pain or accurately localizing discomfort. Clinical observations suggest that many ASD patients may not present with physical symptoms of an illness that most physicians have been taught to recognize in typically developing persons, which further complicates the physicians' diagnostic efforts. In fact, many "autistic behaviors," such as aggression and self-injurious activity, have been attributed to be just "part of the autism." As a result, the ASD patient is frequently referred for behavioral management and/or psychopharmacological intervention because of failure to interpret these behaviors as indicators of pain and discomfort, suggesting an underlying medical condition.

The importance of identifying comorbid, health-related medical conditions associated with ASD are multiple. First, many of these medical conditions are treatable, and if identified and managed, will result in an improved sense of well being, more effective participation in educational and therapeutic programs, and improved quality of life for the patient and his family. Second, identification of medical disorders involving specific organ systems may help us to identify phenotypic and genetic clusters of ASD persons, thus possibly defining meaningful subtypes that may result in a better understanding of subsets of causative and biological mechanisms. However, at this point in time there are few, well-designed population studies that have carefully examined associated medical disorders in autism, and much of the "data" available stems from clinical observations and anecdotal information involving relatively small groups of subjects. In 2003, the Autism Treatment Network (ATN) was established under the guidance of the Northwest Autism Foundation in Portland, Oregon, with the goal of creating a network of academically based multidisciplinary centers that would develop mutually agreed on protocols to begin assessing the prevalence, presenting features, and treatment of comorbid medical conditions across the autism spectrum. Today, the ATN is comprised of fourteen academic institutions across the United States and Canada. With the involvement of the ATN, and possibly other multi-site consortium groups, it is hoped that we may be able to look more carefully at a number of comorbid medical conditions that may have broad implications if identified and treated, resulting in improved behavior, developmental progress, and quality of life, as well as providing leads into potential subsets of ASD that may guide genetic and biologic research.

Space does not allow for a detailed description of the multiplicity of possible medical conditions that may affect a person with autism. Suffice it to say that ASD individuals can and do have many of the same medical disorders and diseases that affect neurotypical persons, although they may present differently. Only some of the more common disorders will be highlighted within this chapter, including gastrointestinal dysfunction, sleep irregularities, metabolic disorders, and hormonal imbalances. However, every primary care and specialty physician and caregiver involved in the care of ASD persons must be alert to the possibility of a wide range of potential medial disorders that could negatively impact the health and behavior of each autistic individual.

\section{SEIZURE DISORDERS}

The prevalence of seizures in adults with autism has been estimated to be between $20 \%$ to $35 \%,{ }^{9}$ and in ASD children the estimate is between $7 \%$ to $14 \%,{ }^{10,11}$ with peak risk periods said to occur during early childhood and adolescence. ${ }^{12}$ All types of seizures can be found in association with ASD, including major motor, myoclonic, and febrile seizures, but complex partial seizures are most frequently reported. $66.7 \%$ of seizures that occur in ASD become apparent after 12 years of age, ${ }^{13}$ with $30 \%$ occurring by 20 years of age. A predilection for seizures appears to be associated with the co-appearance of low cognitive ability, dysmorphic features, and motor impairment, and a long list of syndromes, including tuberous sclerosis and Rett's syndrome. It is likely that the co-occurrence of ASD and seizures may reflect a shared underlying neurobiology.

The clinical identification of seizures may be difficult in some cases, especially partial complex seizures, which can be largely complicated by the presence of atypical body movements and behavioral patterns often seen in association with ASD. Not all body movements or mannerisms observed in ASD are seizure related and may be a manifestation of other medical conditions, such as gastroesophageal reflux disease (GERD). ${ }^{14}$ Further complicating the diagnosis is a lack of direct correlation between electroencephalographic (EEG) activity and clinical seizures. ${ }^{9}$ However, any behavior such as staring 
spells, cessation of activity, eye fluttering, or eye deviation to one side, as well as behavioral changes associated with confusion or followed by fatigue or sleep should raise the suspicion of complex partial seizures, and should lead to further investigation. Obtaining a highquality EEG, especially in toddlers and young children, can be difficult but is achievable. A prolonged overnight EEG can often be helpful, as well as the use of a videotape review of the events recorded at home and/or at school.

Treatment approaches have expanded substantially over the past several years, and a number medication options are available for intervention. In a small number of cases, seizure control can be difficult to achieve and may require the use of multiple medications and possibly the use a vagal nerve stimulator. For the most part, seizure management can be successful in the majority of ASD children, adolescents, and adults with good medical oversight.

In recent years, there has been a growing interest in the possible role of EEGs in autism, with or without the presence of epilepsy. Rates of these abnormal EEGs have been reported to be as high as $60 \%$, and it has been suggested by some investigators that these abnormalities may play a causal role in autism symptomatology. ${ }^{15}$ The significance of epileptiform EEGs in the absence of clinical seizures remains controversial, and as of yet there is no solid data that exists as to their relevance or treatment implications. However, this is an important area for future research. Although these EEG findings may simply be a reflection of the underlying neuropathology responsible for autism, without implication for treatment, it is also possible that individuals with these abnormalities might potentially benefit from intervention. Large population studies correlating EEG abnormalities with autism phenotypes and clinical medication trials with a welldescribed baseline and outcome data could help to begin to answer these questions.

\section{SLEEP DISORDERS}

Sleep problems are highly prevalent in ASD children and rank as one of the most common concurrent clinical disorders. ${ }^{16}$ Prevalence rates vary widely, ranging from $40 \%$ to $80 \%,{ }^{17,18}$ as compared with that of typically developing children in which prevalence rates are approximately $30 \% .{ }^{19}$ In addition, sleep disorders are reported to be more severe in ASD. Among the sleep problems most commonly reported, sleep onset, sleep maintenance, and sleep duration are consistently the most predominant concerns expressed by parents of children with an ASD. ${ }^{20}$ However, other types of sleep disturbances have also been reported in ASD children, including parasomnias (nightmares, screaming), sleeprelated breathing disorders, sleep-related movement dis- orders, and circadian rhythm sleep disorders. Although the frequency of night-time awakening is similar to that of typically developing children, ASD children experience more lengthy periods of night waking (i.e., frequently as much as 2 to 3 hours per night). During this time, the child may laugh, talk, or get up and play with toys/objects in the room, which is a pattern that is not typical of other children with developmental disabilities or typically developing children. ${ }^{21}$

It is known that disordered sleep affects daytime health and can result in neurocognitive dysfunction and behavioral disruptions. In typically developing children, sleep disruption may lead to daytime sleepiness and may manifest itself as hyperactivity, inattention, or aggression. $^{22}$ Thus, sleep disorders can have wide ranging implications for daytime functioning, developmental progress, and quality of life for both the child and his family. Effective intervention can have a positive impact on educational and developmental progress, as well as community life opportunities.

Although sleep difficulties are common in ASD, it is unclear why this dysfunction is so prevalent in this disorder. A number of hypotheses have been proposed. It has been suggested that sleep problems could be the result of 1) intrinsic biological or genetic abnormalities that alter brain structure and/or chemistry, 2) psychological or behavioral disorders associated with the core features of ASD, or 3) factors in the home or environment that are not conducive to good sleep. Any one or a combination of these factors could contribute to sleep disturbances in ASD. ${ }^{21}$

Biological mechanisms may involve the role of melatonin, a neurohormone produced from serotonin in the pineal gland and secreted during night-time hours. Its major role appears to be to organize circadian rhythms, including sleep-wake and core body temperature cycles. Sleep problems in ASD may be related to circadian rhythm dysregulation, which may cause the nervous system to fluctuate causing fluctuation in faulty transmission of entrainment cues, along with social and environmental factors and possible intrinsic biological abnormalities. ${ }^{21}$ This faulty transmission of entrainment cues may be related to an abnormal expression of one or more of 10-clock genes that are believed to form the core of the biological clock, thereby controlling circadian rhythm. Thus, it has been speculated that sleep disorders in ASD may be related to genetic abnormalities associated with melatonin synthesis and its role in modulating synaptic changes. To date, there have been few studies exploring the role of melatonin in sleep pattern abnormalities associated with ASD, but existing data suggests that further investigations are warranted.

Although biological factors may form the basis of many of the sleep disturbances in ASD, co-morbid psychopathology may also be playing a role, at least in some 
cases, especially anxiety, depression, stress, and mood disorders. Symptoms of attention deficit hyperactivity disorder may also play a role and contribute to sleeplessness, as well as sleep disordered breathing, obstructive apnea, respiratory compromise secondary to enlarged tonsils and adenoids, parasomnias, and a variety of medications, including corticosteroids, stimulants, some anticonvulsants, and serotonin reuptake inhibitors, or asthma medications. ${ }^{18}$

It has been recommended that all ASD children be screened for potential sleep disturbances, given the high prevalence rate in this population. ${ }^{18}$ A number of questionnaires can be used including the Children's Sleep Habits Questionnaire (CSHQ) and the Pediatric Sleep Questionnaire (PSQ). Each of these questionnaires explores possible obstructive sleep apnea, restless leg syndrome, behaviors suggestive of parasomnias (night terrors, sleep walking), or symptoms that might suggest nocturnal seizures. Depending on the responses to the questionnaires, referrals to a sleep specialist or a neurologist may be indicated. Further studies may be needed to clarify the nature and cause of the presenting behaviors, including the use of actigraphy and polysomnography. Gastrointestinal disorders, such as GERD, should also be considered as a possible cause of nighttime awakenings and may warrant a referral to a gastroenterologist. ${ }^{23}$

When considering treatment interventions, most sleep specialists recommend the implementation of establishing a program to improve sleep hygiene. This approach seeks to correct problematic behavior and promote regulated and predictable bedtime routines. Behavioral interventions can often be useful in treating children with ASD and may be effective in dealing with problems associated with sleep onset and frequent awakenings.

Pharmacologic interventions may also be indicated. Currently, there is growing evidence in support of the use of melatonin, which is considered a nutritional supplement, and as such, it can be obtained over-the-counter and is relatively inexpensive. Studies have shown that this product can be efficacious in promoting sleep and appears to have no long-term adverse side effects. However, melatonin has not been rigorously studied with regard to dosage, safety, and efficacy, and further studies are needed.

Similarly, there is little data to support the use of other medications for use in promoting and sustaining sleep in ASD. Clonidine has been found to be effective in reducing problems with sleep onset and night-time awakenings in some studies. ${ }^{16}$ Mediations designed to treat anxiety and depression can also be considered, such as mirtazapine and trazodone, fluvoxamine, or clomipramine, have been used in some cases in which obsessional disorders appear to be a factor. Some anticonvulsant medications with sedating properties may also be useful. ${ }^{18}$ It is important, however, that whatever medications are implemented, management be carefully monitored by a sleep specialist or psychopharmacologist with expertise in treating ASD children, because dosage and potential side effects merit cautious and careful implementation and supervision.

\section{GASTROINTESTINAL DISORDERS}

The evaluation of abdominal pain and discomfort in an individual with impaired communication skills and possible dysfunction in the processing of sensory information can be a challenging task. Although many patients present with the easily recognized symptoms of vomiting, diarrhea, and constipation, a substantial number of individuals do not provide these clues. Currently there are no reliable signs or symptoms that can consistently aide the primary care physician to distinguish between functional and organic disorders. Many ASD individuals may express their physical discomfort by manifesting atypical behaviors, such as putting pressure on their abdomen, tapping on their chest, acting aggressively toward others, or demonstrating self-injurious behavior. ${ }^{23}$

At the present time, the true prevalence of gastrointestinal (GI) disorders in ASD is largely unknown, with estimations ranging from $9 \%$ to $70 \%$ or higher. ${ }^{24}$ Evidence-based guidelines for the evaluation of GI symptoms are not yet available for individuals with ASD. However, recently, 8 experienced gastroenterologists reviewed the current literature and published guidelines regarding GI symptoms that frequently occur in the general pediatric population. Based on this information and their collective expertise, they adopted guidelines for the current best practices related to the diagnosis and treatment for children with ASD. These guidelines deal with chronic constipation, abdominal pain, chronic diarrhea, and symptoms of GERD. In addition, these guidelines also raise awareness of the association of GI disorders with self-injurious behavior and sleep disturbances, and the frequent atypical presentation of GI dysfunction in individuals with ASD. ${ }^{22,24}$

Although some ASD persons present with symptoms that are easily recognized by most healthcare providers, many others may not. As previously noted, atypical behaviors may include chest tapping, facial grimacing, and intermittent gulping, chewing on nonedible objects, including shirts and sleeves, constant eating or drinking, seeking of abdominal pressure, aggressions, and selfinjurious behaviors to name just a few. ${ }^{25}$ It is well known that developing children may typically present with behavioral disruptions when not feeling well, and there is little reason to believe that ASD children should act differently. It is also well established that if an individual is ill, he or she is less likely to perform well at school, in a job, or in daily life. Thus, the identification and treatment of medical conditions, including GI disorders, may 
have a positive impact on the ability of the ASD person to participate in programs and therapies and to make meaningful progress.

A number of GI disorders have been reported in association with ASD, including GERD, gastritis, esophagitis, inflammatory bowel disease, celiac disease, Crohn's disease, and colitis. Evaluation of a possible GI disorder should consist of a thorough medical history and physical examination, including anal inspection and examination and assessment of the back and spine for a dimple or tuft of hair. Studies may include the analysis of a stool specimen for parasites and enteric pathogens, stool guaiac, electrolytes/osmolarity, and might include serum electrolytes, liver function tests, assessment of nutritional status, and an abdominal roentgenogram to assess bowel gas pattern and possible retention of stool. ${ }^{23}$ Depending on the results of these assessments and the outcome of attempted medical management and interventions, endoscopy, and/or colonoscopy may be warranted for diagnostic and treatment purposes.

It is not yet known whether GI disorders are more common in the ASD population than in typically developing persons, or whether these disorders should be treated in the same manner in both groups. Similarly, it is not known whether GI dysfunctions are inherent to ASD in some cases or associated phenomena. Welldesigned, well-controlled diagnostic and clinical studies in sizeable populations (probably involving different age groups) are needed to establish evidence-based guidelines for identification and management. Recently, Campbell et al. ${ }^{26}$ reported that disrupted MET gene signaling may contribute to an increased risk for ASD that includes familial GI dysfunction. A functional variant in the promoter of the gene encoding the MET receptor tyrosine kinase has been associated with ASD, and MET protein expression has been found to be decreased in the temporal lobe cortex in ASD postmortem brain. MET is a pleotrophic receptor that is known to be important for brain development, as well as GI repair and motility. This study suggests that by carefully investigating ASD individuals and their families medically, as well as behaviorally, we may be able to begin to identify genetically important subgroups within the larger ASD population. Thus, the identification of medical conditions in ASD persons, in this case, GI disorders, may not only impact the general health and well-being of the patients, but it may lead to improved and more precise definitions of phenotypic and genetic subtypes within this complex heterogeneous disorder.

\section{METABOLIC DISORDERS}

Metabolic disorders are considered to be relatively rarity among individuals with neurodevelopmental disorders. Among this population, the estimated diagnostic yield has been found to vary between $1 \%$ to $2.5 \% .{ }^{27}$ In a more recent study, Engbers et al. ${ }^{28}$ performed repeated metabolic analyses on a cohort of 433 subjects with neurodevelopmental impairments, whose initial evaluations were said to be unremarkable. Of these subjects, 12 (2.8\%) were found to have diagnostic disorders, some of which were treatable. Thus, although metabolic disorders may be rare, it is important to pursue carefully planned diagnostic studies so as to identify potentially manageable disorders. The prevalence of metabolic disorder in ASD is largely unknown.

A number of genetic and metabolic disorders have been associated with ASD, often described in a small number of subjects. In the past, phenylketonuria was often reported to be associated with autistic features, but due to newborn screening, it is less commonly observed. Autistic features have also been reported in association other metabolic disorders, including creatine deficiency syndromes, ${ }^{29}$ urea cycle disorders, ${ }^{30}$ Wilson's disease, and Lesch-Nyhan syndrome. ${ }^{31}$ Space does not allow for a full review of many of these conditions. However, there has been an increasing interest in two of these disorders in particular, and these will be highlighted here in more detail.

There is a growing body of suggestive evidence that mitochondrial dysfunction may play a role in at least a subset of individuals with ASD. Mitochondria perform four central functions in the cell that are relative to the pathophysiology of disease: 1) they provide most of the energy to a cell in the form of ATP, 2) they generate and regulate reactive oxygen species, 3 ) they buffer cytosolic calcium, and 4) they regulate apoptosis through mitochondrial permeability transition pore. ${ }^{32}$ Mitochondrial disorders are heterogeneous and are often multi-systemic. Mitochondria provide much of the energy to the cell, and thus preferentially affect tissues with high-energy demands including the brain, the muscles, the heart, and the endocrine system. However, any organ system can be involved. It has been hypothesized that mitochondria may be a mediator of many common metabolic disorders. Mitochondrial disorders can be caused by genetic defects in mtDNA or nuclear DNA gene that encodes a mitochondrial protein or structural RNAs. Mitochondrial disorders are believed to be rare, with those related to mtDNA estimated to have a frequency of 1 in 5000, and known pathogenic mtDNA mutations detected in cord blood in 1 in 200 live births. The frequency of nDNA mutations affecting mitochondrial genes may be more common than mtDNA mutations. ${ }^{32}$

At the present time, the prevalence of mitochondrial disorders in ASD is largely unknown. In 2005, Oliveira et al. ${ }^{33}$ published a population-based study, which was a survey of school-aged children with ASD. The investigators found that $7 \%$ of their subjects met criteria for a definite mitochondrial respiratory chain disorder, the af- 
fected children being clinically indistinguishable from ASD children without a mitochondrial disorder. In a more recent study, Weissman et al. ${ }^{34}$ reviewed the medical records of 25 ASD children with muscle biopsy proven enzyme-defined or mutation-defined mitochondrial electron transport chain dysfunction. Clinically, 24 of the 25 subjects demonstrated one or more abnormalities uncommon in ASD including cardiovascular, endocrine, and hematologic findings. There were 19 subjects exhibiting constitutional symptoms, such as low physical endurance, repeated regressions after the age of 3 years of age, delayed gross and fine motor milestones, and the involvement of multiple organ systems. Abnormalities were found in blood lactate, plasma alanine, and serum ALT and AST in 76\%, 36\%, and 52\%, respectively. No differences were noted between males and females. The most common findings were deficiencies in complex I (64\%) and complex III (20\%). Thus, although all children carried a diagnosis of idiopathic autism, detailed clinical and biochemical evaluation identified features that appeared to differentiate these children from others with essential autism. The authors concluded that with careful clinical and biochemical analysis, ASD children with co-occurrence of mitochondrial dysfunction can be distinguished from those with idiopathic ASD, and that those with mitochondrial autism may comprise a significant subset of individuals with autism. If this hypothesis is true, this subset should be seen as genetically different from ASD children without a mitochondrial dysfunction. Whether the mitochondrial dysfunction in these cases is primary (causative) or secondary (an associated phenomenon) remains to be determined, and also opens this important question to further research.

Smith-Lemli-Opitz syndrome (SLOS) is another metabolic disorder that has been frequently associated with features of ASD. ${ }^{35}$ SLOS is known to be an autosomal recessive disorder associated with an inborn error of cholesterol synthesis caused by mutations of the 7-dehydrocholesterol reductase gene (DHCR7) located on chromosome $11 \mathrm{q} 12-13 .^{36}$ The syndrome has an estimated incidence of 1 in 20,000 to 1 in 60,000 births and a carrier frequency of $1 \%$. Cholesterol is essential for neuroactive steroid production, growth of myelin membranes, and normal embryonic and fetal development. In addition, it is believed to modulate oxytocin receptors, ligand activity and G-protein coupling of the serotinin-1A receptor. ${ }^{37}$ It has been hypothesized that a deficit in cholesterol may disrupt these biological mechanisms, thereby contributing to clinical features of ASD seen in SLOS. It has been estimated that $50 \%$ to $75 \%$ of individuals with SLOS may meet criteria for ASD.

Clinically, although physical features may not be readily recognizable in mild cases, individuals with SLOS frequently exhibit dysmorphic features associated with ptosis, upturned nares, small chin, bi-temporal nar- rowing, microcephaly and webbing between the second and third toes. Additional characteristics include developmental delay, hyperactivity, irritability, temper tantrums, aggressions, and self-injury. The diagnosis of SLOS can be confirmed by the presence of an elevated level of plasma 7-dehydrocholesterol relative to the cholesterol level. Identification of this disorder is important, given the fact that it is one cause of ASD that is partially treatable with cholesterol supplementation.

\section{HORMONAL DYSFUNCTION}

There has been an unwritten lore in the world of autism that when children with ASD go through adolescence, then their behavior worsens. This change in behavior has usually been attributed to the hormonal changes associated with the teenage years, but no formal studies have been reported to support this hypothesis. Hormonal changes could also be reflected in association with precocious puberty, accelerated, or reduced physical growth, and/or behavioral disruptions associated with menstrual pain or discomfort. An additional disorder that should be considered is one of congenital adrenal hyperplasia that we have diagnosed in three adolescent girls with ASD. ${ }^{38}$ Some of these factors have been reviewed in a study published by Carr et al., ${ }^{39}$ in which both physical discomfort and pain associated with the menstrual cycle was cited as a possible cause of problem behaviors in adolescent girls. The authors also raise the possibility that fluctuations in progesterone and estrogen levels during adolescence may be an important variable. Abnormalities in progesterone and/or estrogen levels have been observed in a subset of behaviorally disruptive adolescent girls with ASD in our clinical program. When this hormonal imbalance is treated, the problem behaviors decline significantly and in some cases, disappear. ${ }^{38}$

This theme has been further expanded in a recent study in which adolescent girls with autism, Down syndrome, and cerebral palsy were evaluated retrospectively regarding gynecological complaints. The girls with autism were significantly more likely to present with behavioral issues than those in the two other groups. Successful management included the use of nonsteroidal anti-inflammatory medications, oral contraceptives, and education..$^{40}$ Thus, there is some evidence that hormonal dysregulation during adolescence may be an underlying factor for some of the behavioral problem observed in some of the ASD teenagers. At this time, there is very limited data regarding hormonal changes in adolescent ASD girls, and there is almost no similar research in ASD teenage boys. This is a very important but significantly understudied topic that merits increased attention to research.

There are numerous other medical conditions that deserve mentioning. These include the growing concern 
regarding the prevalence of osteoporosis among individuals with ASD and the possible role of self-imposed dietary selection, and/or the use of special diets that may restrict calcium and vitamin D as part of treatment programs. ${ }^{41}$ Obesity is also becoming a growing concern, not only among ASD individuals but in regard to the whole population within the United States. In ASD, contributing factors may include the use of food re-inforcers as part of a behavioral program, the preference for carbohydrates shown by many ASD persons, the use of some medications (such as risperidone), and the lack of physical exercise and activity. ${ }^{42}$ Additional medical conditions that merit scrutiny include recurrent otitis media, hearing impairment, bladder and renal disorders, hypertension, diabetes, allergies, recurrent headaches/migraine, dental pain, immune disorders, and bacterial and viral illnesses, just to name a few. Any or all of these can result in significant behavior alterations. Much research is needed to assess the prevalence of these and other intercurrent illnesses in ASD, their mode of presentation, and whether medical management should be the same or different from that of typically developing individuals. We have much to learn in regard to organ systems in ASD outside of the brain, and how this information may contribute to a better understanding of the biological processes that underlie this disorder throughout a patient's lifespan.

ASD has been traditionally seen as a disorder primarily involving atypical neurodevelopment in the brain. Very little attention has been paid to other organ systems. It is becoming increasingly apparent that many ASD individuals may have associated medical conditions, which up to this point have gone unrecognized, and which are now only just becoming evident. At this point in time, there are very few, if any, large population studies related to the investigation of potential comorbid medical conditions in individuals with autism. Thus, there is little information regarding clinical presentation, which could guide the primary care physician, prevalence rates, effective modes of treatment, or developmental or behavioral outcomes as the result of diagnosis and management. Research designed to begin to address these questions is a major part of the agenda of the ATN and other clinical collaboratives. It is likely that the recognition of many of these medical conditions followed by appropriate treatment may result in better, more effective participation in and benefit from therapies and interventions as the result of improved health, and ultimately improved developmental outcomes. In addition, some of these medical conditions may begin to help us subcategorize specific populations within the larger ASD framework, resulting in more specific clinical and biological phenotyping and genotyping of ASD patients throughout their lifespan. More research is needed involving organ systems outside the brain. The information to be obtained from these investigations may broaden our understanding of the interplay between these systems, thereby expanding our knowledge of the underling biologic mechanisms involved in the autism spectrum disorders.

\section{CONCLUSIONS}

It is becoming increasingly evident that many ASD children, adolescents, and adults can and often do experience a number comorbid medical conditions, the nature and prevalence of which remain as yet to be poorly defined. Many of these disorders have been largely ignored, at least in part, due to the challenges involved in conducting a meaningful medical history and physical examination in a frequently nonverbal patient whose behavior may interfere with a detailed assessment. However, there is a growing importance in identifying these disorders. First, ASD individuals deserve the same highquality healthcare available to their neurotypical peers. They deserve to have treatable medical conditions identified and appropriately treated, resulting in decreased discomfort and improved overall health, thus allowing them to better participate in their therapeutic, educational, and vocational programs, and to achieve their best potential. Second, by identifying associated medical conditions and including them as part of the clinical phenotype, we may be able to better clinically subtype groups of ASD individuals, thus leading to more accurate genotypic identification. Much progress has been made during the past 5 to 10 years, but much remains to be learned. With advancing technology and improved diagnostic measures, it is anticipated that our understanding of the ASDs will improve and expand, resulting in more targeted and effective interventions in the future.

\section{REFERENCES}

1. Kanner L. Autistic disturbances of affective contact. Nervous Child 1943;2:217-50.

2. American Psychiatric Association Diagnostic and Statistical Manual of Mental Disorders, 4th edit (DSM-IV). Washington DC: APA, 1994.

3. Newschaffer CJ, Croen LA, Daniels J, et al. The epidemiology of autism spectrum disorders. Annu Rev Public Health 2007;28:235258.

4. Ming X, Brimacombe M, Wagner GC. Prevalence of motor impairment in autism spectrum disorders. Brain Dev 2007;29:565570 .

5. Lainhart JE, Bigler ED, Bocian M, et al. Head circumference and height in autism: a study by the Collaborative Program of Excellence in Autism. Am J Med Genet A 2006;140:2257-2274.

6. Landa RJ, Garrett-Mayer E. Development of infants with autism spectrum disorders: a prospective study. J Child Psychol Psychiatry 2006;47:629-638.

7. Landa RJ. Diagnosis of autism spectrum disorders in the first 3 years of life. Nat Clin Pract Neurol 2008;4:138-147.

8. Lauritsen MB, Pedersen CB, Mortensen PB. Effects of familial risk factors and place of birth on the risk of autism: a nationwide register-based study. J Child Psychol Psychiatry 2005;46:963-971.

9. Minshew NJ, Sweeney JA, Bauman, ML. Neurologic aspects of autism. In: Cohen DJ, Volkmar FR, eds. Handbook of autism and 
pervasive developmental disorders, 2nd ed. New York: Wiley, 1997:344-369.

10. Rapin I. Historical data. In: Rapin I, ed. Preschool children with inadequate communication: developmental language disorder, autism, low IQ. London: MacKeith, 1996:98-122.

11. Tuchman RF, Rapin I, Shinnar S. Autistic and dysphasic children. II: Epilepsy. Pediatrics 1999;88:1219-1225.

12. Volkmar FR, Nelson DS. Seizure disorders in autism. J Amer Acad Child Adolesc Psychiatry 1990;29:127-129.

13. Rossi PG, Parmeggian A, Bach V, Santucci M, Visconti P. EEG features and epilepsy in patients with autism. Brain Dev 1995;17: 169-174.

14. Buie T. Gastrointestinal issues encountered in autism In: Bauman ML, Kemper TL, eds. The neurobiology of autism. Johns Hopkins University Press: Baltimore, 2005:103-117.

15. Spence SJ, Schneider MT. The role of epilepsy and epileptiform EEGs in autism spectrum disorders. Pediatr Res 2009;65:599606.

16. Ming $X$, Brimacombe $M$, Chaaban J, et al. Autism spectrum disorders: concurrent clinical disorders. J Child Neurol 2008; 23:6-13.

17. Richdale AL. Sleep problems in autism: prevalence, cause and intervention. Dev Med Child Neurol 1999;41:60-66.

18. Johnson KP, Giannotti F, Cortesi F. Sleep patterns in autism spectrum disorders. Child Adolesc Psychiatric Clin N Am 2009;18: 917-928.

19. Ferber R. Childhood sleep disorders. Neurol Clinics 1996;14:493511.

20. Malow BA, Marzec ML, McGrew SG, Wang L, Henderson LM, Stone WI. Characterizing sleep in children with autism spectrum disorders: a multidimensional approach. Sleep 2006;28:15591567.

21. Richadale AL, Schreck KA. Sleep problems in autism spectrum disorders: prevalence, nature and possible biopsychosocial aetiologies. Sleep Med Rev 2009;13:403-411.

22. Owens J, Opipari L, Nobile C, Spirito A. Sleep and daytime behavior in children with obstructive apnea and behavioral sleep disorders. Pediatrics 1998;102:1178-1184.

23. Buie T, Fuchs GJ, Furuta GT, et al. Recommendations for evaluation and treatment of common gastrointestinal problems in children with ASDs. Pediatrics 2010;125(suppl):S19-S29.

24. Buie T, Campbell DB, Fuchs GJ, et al. Evaluation, diagnosis and treatment of gastrointestinal disorders in individuals with ASDs: a consensus report. Pediatrics 2010;125(suppl):S1-S18.

25. Buie T. Clinical presentation of gastrointestinal disorders in nonverbal ASD children. Unpublished data, 2009.

26. Campbell DB, Buie TM, Winter $\mathrm{H}$, et al. Distinct genetic risk based on association of MET in families with co-occurring autism and gastrointestinal conditions. Pediatrics 2009;123:1018-1024.

27. Von Karnebeck CD, Jansweijer MC, Leenders AG, et al. Diagnos- tic investigations in individuals with mental retardation: a systematic literature review of their usefulness. Eur J Hum Genet 2005;13:6-25.

28. Engbers HM, Berger R, van Hasselt P, et al. Yield of additional metabolic studies in neurodevelopmental disorders. Ann Neurol 2008;64:212-217.

29. Nasrallah F, Feki M Kaabachi N. Creatine and creatine deficiency syndromes: biochemical and clinical aspects. Ped Neurol 2010;42: $1-13$.

30. Gorker I, Tuzun O. Autistic-like findings associated with a urea cycle disorder I a 4-year old girl. J Psychiatry Neurosci 2005;30: 133-135.

31. Trauner DA. Cognitive and behavioral manifestations of metabolic and chromosomal disorders. In: Frank Y, ed. Pediatric behavioral neurology. New York: CRC Press, 1996:251-268.

32. Wallace DC, Fan W, Procaccio V. Mitochondrial energetics and therapeutics. Annu Rev Pathol Mech 2010;5:297-348.

33. Oliveira G, Diogo L, Grazina M, et al. Mitochondrial dysfunction in autism spectrum disorders: a population-based study. Dev Med Child Neurol 2005;47:185-189.

34. Weissman JR, Kelley RI, Bauman ML, et al. Mitochondrial disease in autism spectrum disorder patients: a cohort analysis. PLos ONE (serial online) 2008;3:e3815. Available at: www.plosone.org. Accessed December 2008.

35. Bukelis I, Porter FD, Zimmerman AW, Tierney E. Smith-LemliOpitz syndrome and autism spectrum disorder. Am J Psychiatry 2007;164:1655-1661.

36. Kelley RI, Hannekam RCH, Smith-Lemli-Opitz syndrome. In: Scriver CR, Beaudet AL, Valle D, Sly WS, eds. The metabolic and molecular basis of inherited disease, 8th edit. New York: McGraw Hill, 2001:6183-6201.

37. Aneia A, Tierney E. Autism: the role of cholesterol in treatment. Int Rev Psychiatry 2008;20:165-170.

38. Herzog A. Hormonal abnormalities may be present in a subset of girls with ASD. Unpublished data, 2008.

39. Carr EG, Smith CE, Glacin TA, Whelan BM, Pancari J. Menstrual discomfort as a biological setting event for severe problem behaviors: assessment and intervention. Am J Mental Retard 2003;108: 117-133.

40. Burke LM, Kalpakjian CZ, Smith YR, Quint EH. Gynecological issues of adolescents with Down syndrome, autism, and cerebral palsy. J Pediatr Adolesc Gynecol 2010;23:11-15.

41. Hediger ML, England LJ, Molloy CA, Yu KF, Manning-Courtney $\mathrm{P}$, Mills JL. Reduced bone cortical thickness in boys with autism or autism spectrum disorder. J Aut Dev Disord 2008;38:848-856.

42. Curtin C, Bandini LG, Perrin EC, Tyboi DJ, Must A. Prevalence of overweight in children and adolescents with attention deficit hyperactivity disorder and autism spectrum disorders: a chart review. BMC Pediatr 2005;5:48-58. 